\title{
Sertindole controlled symptoms of schizophrenia without motor side effects
}

\author{
Zimbroff DL, Kane JM, Tamminga CA, et al, and the Sertindole Study Group. Controlled, dose-response study of sertindole and haloperidol \\ in the treatment of schizophrenia. Am J Psychiatry 1997 Jun;154:782-91.
}

\section{Objective}

To determine the safety and efficacy of sertindole compared with haloperidol and placebo in patients with schizophrenia.

\section{Design}

8 week randomised, double blind, placebo controlled trial.

\section{Setting}

43 psychiatric inpatient centres; 41 in the United States and 2 in Canada.

\section{Patients}

497 patients who were 18 to 65 years of age (mean age 39 y, $78 \%$ men) and had a primary diagnosis of Diagnostic and Statistical Manual of Mental Disorders (DSM), 3rd edition, revised or DSM-IV schizophrenia, no other primary psychiatric diagnoses, and a combined score of $\geqslant 8$ on any 2 of the positive symptoms of the Brief Psychiatric Rating Scale (BPRS). Exclusion criteria were tardive dyskinesia, substance abuse, or confounding medical or neurological problems. 477 patients $(96 \%)$ were included in the intention to treat analysis.

\section{Intervention}

If the total BPRS score did not improve $>20 \%$ and scores on each item of the Abnormal Involuntary Movement Scale were $<3$ during a 4 to 7 day placebo lead in period, patients were allocated to 1 of 7 treatment groups each lasting 8 weeks: placebo $(\mathrm{n}=73)$; sertindole, $12 \mathrm{mg} /$ day $(\mathrm{n}=76) ; 20 \mathrm{mg} /$ day $(\mathrm{n}=68) ; 24 \mathrm{mg} /$ day $(\mathrm{n}=72) ;$ haloperidol, $4 \mathrm{mg} /$ day $(\mathrm{n}=71) ; 8$ $\mathrm{mg} /$ day $(\mathrm{n}=67)$; or $16 \mathrm{mg} /$ day $(\mathrm{n}=70)$.

\section{Main outcome measures}

Changes in scores on psychiatric rating scales (Positive and Negative Syndrome Scale [PNSS], 18 item BPRS, Scale for the Assessment of Negative Symptoms [SANS], and Clinical Global
Impression Scale [CGIS]). Extrapyramidal symptoms were assessed by changes in scores on movement rating scales, presence of adverse events, and need for medications.

\begin{abstract}
Main results
All doses of sertindole were more effective than placebo for total scores on the PNSS, the BPRS, and the CGIS. Haloperidol was also more effective than placebo with the exception of $4 \mathrm{mg} /$ day of haloperidol on the CGIS. Scores on the positive subscale of the PNSS were reduced compared with placebo for sertindole, $20 \mathrm{mg} /$ day ( $\{95 \%$ CI for the 4.8 mean decrease in score 2.1 to $7.5\}^{*}, \mathrm{p}<0.001$ ) and $24 \mathrm{mg} /$ day ( $\{\mathrm{CI}$ for the 3.2 mean decrease in score 0.7 to 5.7$\}^{*}, \mathrm{p} \leqslant 0.03$ ) and for all 3 doses of haloperidol $(p \leqslant 0.04)$. For negative symptoms only, $20 \mathrm{mg}$ of sertindole was superior to placebo on the SANS. The sertindole and haloperidol groups did not differ. Changes in extrapyramidal symptoms scores did not differ for patients who received sertindole compared with placebo. All doses of haloperidol produced more motor side effects than placebo or sertindole. Adverse events and number of patients requiring medication for extrapyramidal symptoms were more common in the haloperidol groups.
\end{abstract}

\section{Conclusions}

Sertindole and haloperidol were more effective than placebo in treating positive symptoms of schizophrenia. Sertindole was associated with fewer extrapyramidal side effects than haloperidol.

*Numbers calculated from data in article.

Source of funding: Abbott Laboratories.

For article reprint:Dr D L Zimbroff, 1317 West Foothill Boulevard, Suite 140, Upland, CA 91786, USA.Fax +19099810639

\section{Commentary}

This large multicentre study by Zimbroff $e t$ $a l$ is of sound methodology and shows that sertindole is as effective as haloperidol but with fewer extrapyramidal side effects. However, some caveats exist regarding the practical clinical application of this work.

Firstly, known treatment resistant patients were specifically excluded from the protocol. New antipsychotics are often tried with patients who have failed to respond to other agents. Although this may be a valid tactic, this study does not support efficacy in such patients.

Secondly, much is made of the effect on negative symptoms. $20 \mathrm{mg}$ of sertindole, led to statistically significant improvement of negative symptoms relative to placebo at several time points, whereas the effect of haloperidol on negative symptoms was statistically significant at only the 5 week time point. The bottom line is, however, that no statistically significant difference was found between the effects of haloperidol and sertindole on negative schizophrenic symptoms and any conclusion that sertindole is better for negative symptoms is not supported by this study.

Thirdly, the effects of sertindole on the electrocardiogram (ECG) are minimised in this study but they are of serious practical consequence. ECG monitoring to measure QT interval is recommended in the prescribing information for sertindole and most psychiatrists would find this to be a burdensome requirement.

Other new antipsychotic agents (eg, risperidone and olanzapine) are available which are as effective as standard drugs but show fewer extrapyramidal side effects. ${ }^{12}$ Most psychiatrists would choose to use 1 of these other agents rather than be troubled by the need for ECG monitoring when no sound evidence exists to support the superior clinical efficacy of sertindole.

Malcolm Peet, MB, ChB, FRCPsych University of Sheffield Sheffield, UK

1 Kennedy E, Song F, Hunter R, et al. Risperidone versus "conventional" antipsychotic medication for schizophrenia. In: the Cochrane Database of Systematic Reviews [updated 01 September 1997]. In the Cochrane Library [database on disk and CD-ROM]. The Cochrane Collaboration; issue 4. Oxford: Update Software; 1997.

2 Tollefson GD, Beasley CM, Tran PV, et al. Olanzapine versus haloperidol in treatment of schizophrenia and schizoaffective and schizophreniform disorders: results of an interphreniform disorders: results of an inter1997;154:457-65.

Editor's note:see also 15 and 17 in this issue of Evidence-Based Mental Health. 